\title{
Space-time Schrödinger symmetries of a post-Galilean particle
}

\author{
Carles Batlle ${ }^{a}$ and Joaquim Gomis ${ }^{b}$ \\ ${ }^{a}$ Departament de Matemàtiques and IOC, Universitat Politècnica de Catalunya, EPSEVG, \\ Av. V. Balaguer 1, E-08808 Vilanova i la Geltrú, Spain \\ ${ }^{b}$ Departament de Física Quàntica i Astrofísica and Institut de Ciències del Cosmos (ICCUB), \\ Universitat de Barcelona, \\ Martí i Franquès 1, E-08028 Barcelona, Spain \\ E-mail: carles.batlle@upc.edu, joaquim.gomis@ub.edu
}

ABSTRACT: We study the space-time symmetries of the actions obtained by expanding the action for a massive free relativistic particle around the Galilean action [1]. We obtain all the point space-time symmetries of the post-Galilean actions by working in canonical space. We also construct an infinite collection of generalized Schrödinger algebras parameterized by an integer $M$, with $M=0$ corresponding to the standard Schrödinger algebra. We discuss the Schrödinger equations associated to these algebras, their solutions and projective phases.

Keywords: Space-Time Symmetries, Classical Theories of Gravity

ARXIV EPRINT: 2004.13636 


\section{Contents}

1 Introduction 1

2 The canonical action of a post-Galilean particle 4

3 Symmetries of post-Galilean particle for $M=1 \quad 5$

4 Generalized Schrödinger algebras $\quad 9$

5 Generalized Schrödinger equation and projective invariance 13

6 Conclusions and outlook $\quad 19$

A Invariance of the contraints under generalized boosts and rotations 20

B Transformation of the canonical variables under the $M$ th level symmetry $\begin{array}{ll}\text { generators } & 20\end{array}$

C Invariance of the constraints under the full set of transformations $\quad 22$

\section{Introduction}

The nonrelativistic gravity theory of Newton is only invariant under the Galilei group. Corrections to the non-relativistic Newtonian theory at higher order in $1 / c$ are famously important for the original experimental evidence for general relativity $[2,3]$. Higher-order (parametrized) post-Newtonian corrections to the Keplerian two-body motion are also of central importance in current investigations of binary gravitational wave sources [4-8]. The effective one-body approach $[5,9]$ was inspired in part by the developments of the classical mechanical relativistic two-body problem [10-13].

Starting from the relativistic Poincaré algebra, one can perform an algebra contraction to the non-relativistic Galilei algebra. Alternatively, starting from the Galilei transformations one can include relativistic corrections at every order in $1 / c$. With corrections up to a finite order, this system has neither Galilei nor Poincaré symmetry. Only when including an infinite set of corrections, with specific coefficients, does one regain the Poincaré symmetry. However, it has recently been argued that the finite-correction-case has a symmetry algebra [1], but this requires enlarging the space on which the transformations act.

It is well known that the most general point symmetries of the Schrödinger equation and the free non-relativistic particle is the Bargmann group [14] with two extra generators, the dilations and the special conformal transformations in one dimension, also called expansions $[15,16]$. Recently, the action and symmetries of a post-Galilean particle, which 
includes corrections of arbitrarily high order in $1 / c$ to the non-relativistic particle in an infinite extended Minkowski space, has been constructed [1]. This study is motivated by the importance of higher-order post-Galilean corrections to the Keplerian two-body motion in current investigations of binary gravitational wave sources [4-8].

The action of a post-Galilean particle in an infinite dimensional Minkowski space with coordinates $\left(t_{(m)}, x_{(m)}^{a}\right), a=1, \ldots, d$, is obtained expanding the action for a massive free relativistic particle $S=-m c \int \mathrm{d} \tau \sqrt{-\dot{X}^{\mu} \dot{X}_{\mu}}$ in powers of $1 / c^{2}$ where the coordinates $X^{\mu}$ are $^{1}$

$$
\begin{aligned}
\frac{1}{c} X^{0} & =t_{(0)}+\frac{1}{c^{2}} t_{(1)}+\frac{1}{c^{4}} t_{(2)}+\ldots, \\
X^{a} & =x_{(0)}^{a}+\frac{1}{c^{2}} x_{(1)}^{a}+\frac{1}{c^{4}} x_{(2)}^{a}+\ldots
\end{aligned}
$$

One gets a series $S_{(0)}+S_{(1)}+S_{(2)}+\ldots$, with $S_{(n)}$ corresponding to power $c^{2-2 n}$. The first contributions are [1]

$$
\begin{aligned}
& S_{(0)}=-m c^{2} \int \mathrm{d} \tau \dot{t}_{(0)}, \\
& S_{(1)}=m \int \mathrm{d} \tau\left(-\dot{t}_{(1)}+\frac{1}{2} \frac{\dot{x}_{(0)}^{2}}{\dot{t}_{(0)}}\right), \\
& S_{(2)}=\frac{m}{c^{2}} \int \mathrm{d} \tau\left(-\dot{t}_{(2)}+\frac{\dot{x}_{(0)}^{a} \dot{x}_{(1) a}}{\dot{t}_{(0)}}-\frac{\dot{t}_{(1)} \dot{x}_{(0)}^{2}}{2 \dot{t}_{(0)}^{2}}+\frac{\dot{x}_{(0)}^{4}}{8 \dot{t}_{(0)}^{3}}\right),
\end{aligned}
$$

where $\dot{x}_{(0)}^{4}=\left(\dot{x}_{(0)}^{2}\right)^{2}$.

As shown in [1], the symmetries of action $S_{(M+1)}, M \geq 0$, realize the algebra [20-22]

$$
\begin{aligned}
{\left[J_{a b}^{n}, J_{c d}^{m}\right] } & =\delta_{c b} J_{a d}^{(n+m)}-\delta_{a c} J_{b d}^{(n+m)}+\delta_{b d} J_{a c}^{(n+m)}-\delta_{b d} J_{a c}^{(n+m)}, \\
{\left[J_{a b}^{n}, B_{c}^{m}\right] } & =\delta_{b c} B_{a}^{(n+m)}-\delta_{a c} B_{b}^{(n+m)} \\
{\left[J_{a b}^{n}, P_{c}^{m}\right] } & =\delta_{b c} P_{a}^{(n+m)}-\delta_{a c} P_{b}^{(n+m)} \\
{\left[B_{a}^{(n)}, P_{b}^{(m)}\right] } & =\delta_{a b} H^{(n+m+1)} \\
{\left[H^{(m)}, B_{a}^{(m)}\right] } & =-P_{a}^{(n+m)} \\
{\left[B_{a}^{(n)}, B_{b}^{(m)}\right] } & =J_{a b}^{(n+m+1)}
\end{aligned}
$$

truncated at level $M$, that is, for $n, m=0,1, \ldots, M$, with any generator with index higher than $M$ set to zero, except for $H^{(M+1)}$ which plays the role of a central charge of the truncated algebra. ${ }^{2}$ The generators $B_{a}^{(0)}$ and $J_{a b}^{(0)}$ generate, respectively, Galilean boosts and ordinary rotations, while $B^{(n)}$ and $J_{a b}^{(n)}$ for $n \geq 1$ are generalized boosts and rotations which transform coordinates between different levels of the expansion in $1 / c^{2}$.

\footnotetext{
${ }^{1}$ The expansion is in powers of $1 / c^{2}$ because we are only interested in symmetries of a free theory in flat space-time and this is how $c$ appears in a post-Galilean expansion of the boosts. In theories that also have gravity, the expansion in $1 / c$ could take into account strong gravity effects, see [17-19].

${ }^{2}$ From the abstract algebra point of view, one could also have the non-central charges $J_{a b}^{(M+1)}$, but they are not realized in the actions $S_{(M+1)}$.
} 
Disregarding the total derivative term $-m / c^{2 M} \dot{t}_{(M+1)}$ which appears in $S_{(M+1)}, M \geq$ 0 , the canonical analysis of the above actions suggest the presence of $M+1$ first class primary constraints [1] and therefore the existence of $M+1$ gauge transformations. For instance, for $M=0$ one has the single constraint

$$
\phi^{(0)}=\frac{1}{2} p_{a}^{(0)} p^{(0) a}-m E^{(0)},
$$

while for $M=1$ there are two constraints ${ }^{3}$

$$
\begin{aligned}
& \phi^{(0)}=p_{a}^{(0)} p^{(1) a}-\frac{1}{2} E^{(1) 2}-\frac{m}{c^{2}} E^{(0)}, \\
& \phi^{(1)}=\frac{1}{2} p_{a}^{(1)} p^{(1) a}-\frac{m}{c^{2}} E^{(1)} .
\end{aligned}
$$

Here $p_{a}^{(0)}$ and $p_{a}^{(1)}$ are the canonical momenta associated to $x_{(0) a}$ and $x_{(1) a}$, respectively, where $E^{(0)}$ and $E^{(1)}$ are minus the canonical conjugated momenta of $t_{(0)}$ and $t_{(1)}$.

The presence of the above $M$ first class constraints means that there are $M$ gauge symmetries. Fixing the gauge does not destroy the space-time invariances of the theory and just introduces a different realization. It should be noticed [1,23] that, if in addition to fixing the gauge, one performs an adequate projection in the space of all the $x$, each of the actions in this sequence yields all the terms of the action obtained by expanding the relativistic action up to the given order.

As is well known [15, 16], for $M=0$ the Galilean algebra can be extended to the Schrödinger algebra adding two new generators, $D$, the generator of space-time dilatations, and $C$, which generates special conformal transformations. Together with the Hamiltonian $H$, they form a $s l(2, \mathbb{R})$ subalgebra which, in the normalization for $D$ that we will use, reads $\{D, C\}=-2 C,\{D, H\}=2 H,\{C, H\}=D$.

In this paper we first construct the explicit form of the $M+1$ of the mass-shell constraints associated to the action $S_{(M+1)}, M \geq 0$. These constraints are first class and allow us to construct the canonical action $S_{M+1}^{c}$. We write then the most general canonical generator $G$ linear in the momenta. The request that $G$ be a constant of motion implies a set of partial differential equations for the unknown functions of the generator $G$ that we solve. ${ }^{4}$ This allows to construct the most general point symmetry transformation of the canonical action $S_{M+1}^{c}$.

The algebra of these transformations is a generalization of the Schrödinger algebra, and does not contain, except for the case $M=0$, an $s l(2, \mathbb{R})$ algebra, and therefore they are not truncations of the Schrödinger-Virasoro algebra [26], and they are also different from the conformal Galilean algebras (CGA) with dynamical exponent $z=2 / N$, with $N$ positive integer, since these contain an $s l(2, \mathbb{R})$ subalgebra [27-30]. The algebras that we obtain in this paper contain the generators $H^{(n)}, B^{(n)}, J_{a b}^{(n)}$ that close under the algebra (1.6) and the new generators $D^{(n)}$, which generalize the dilatations, and a single generator $C$ of expansions.

\footnotetext{
${ }^{3}$ Here and later on, the constraints and the generators that we will define should be understood as having an additional subscript denoting the value of $M$. We prefer not to write it in order to not to charge the notation too much. Since all the computations are done for fixed $M$, this should not cause any confusion.

${ }^{4}$ For the use of this method in non-relativistic systems see for example [24, 25] and references therein.
} 
We consider the $M+1$ Schödinger equations associated to the quantization of the postGalilean particle described by the action $S_{M+1}^{c}$. We also study the projective character of the wave function.

The paper is organized as follows. In section 2 we write the canonical action $S_{M+1}^{c}$ for arbitrary $M$. In section 3 we deal with the $M=1$ case, for which we compute the most general point transformation by means of the canonical version of Noether's theorem and obtain the first extended Schrödinger algebra. Section 4 contains our main results, and we present the algebra for arbitrary $M$. The Schrödinger equations associated to the new algebras and their projective invariance are discussed in section 5. Our results are summarized in section 6, and some open problems are discussed. Appendix A contains the proof of the invariance of the constraints under generalized boosts and rotations proposed in section 2, appendix B lists the transformations of all the canonical variables, and finally appendix $\mathrm{C}$ discusses the invariance of the constraints under the full set of transformations.

\section{The canonical action of a post-Galilean particle}

In this section we construct the canonical action $S_{M+1}^{c}$ associated to the post-Galilean action $S_{(M+1)}$ for a generic $M$, written in terms of the mass-shell constraints $\phi^{(k)}$

$$
S_{M+1}^{c}=\int d \tau \sum_{k=0}^{M}\left(-E^{(k)} \dot{t}_{(k)}+p_{a}^{(k)} \dot{x}_{(k)}^{a}-e_{(k)} \phi^{(k)}\right) .
$$

The constraints $\phi^{(k)}$ are the generalization of those for $M=0$ and $M=1[1,23]$. Explicitly, the $M+1$ constraints corresponding to level $M$ are

$$
\phi^{(k)}=-\frac{m}{c^{2 M}} E^{(k)}+\frac{1}{2} \sum_{l=k}^{M} p_{a}^{(l)} p^{(M+k-l) a}-\frac{1}{2} \sum_{l=k}^{M-1} E^{(l+1)} E^{(M+k-l)},
$$

for $k=0,1, \ldots, M$. They reproduce (1.7) for $M=0$ and (1.8) for $M=1$, and for the next few $M$ they yield

$$
\begin{aligned}
\phi^{(0)} & =p_{a}^{(0)} p^{(2) a}+\frac{1}{2} p_{a}^{(1)} p^{(1) a}-E^{(1)} E^{(2)}-\frac{m}{c^{4}} E^{(0)}, \\
\phi^{(1)} & =p_{a}^{(1)} p^{(2) a}-\frac{1}{2} E^{(2) 2}-\frac{m}{c^{4}} E^{(1)}, \\
\phi^{(2)} & =\frac{1}{2} p_{a}^{(2)} p^{(2) a}-\frac{m}{c^{4}} E^{(2)} .
\end{aligned}
$$

for $M=2$ and

$$
\begin{aligned}
\phi^{(0)} & =p_{a}^{(0)} p^{(3) a}+p_{a}^{(1)} p^{(2) a}-\frac{1}{2} E^{(2) 2}-E^{(1)} E^{(3)}-\frac{m}{c^{6}} E^{(0)} \\
\phi^{(1)} & =p_{a}^{(1)} p^{(3) a}+\frac{1}{2} p_{a}^{(2)} p^{(2) a}-E^{(2)} E^{(3)}-\frac{m}{c^{6}} E^{(1)} \\
\phi^{(2)} & =p_{a}^{(2)} p^{(3) a}-\frac{1}{2} E^{(3) 2}-\frac{m}{c^{6}} E^{(2)} \\
\phi^{(3)} & =\frac{1}{2} p_{a}^{(3)} p^{(3) a}-\frac{m}{c^{6}} E^{(3)}
\end{aligned}
$$


for $M=3$. Notice that the last $M$ constraints for level $M$ coincide in form with the $M$ constraints from level $M-1$, with different variables, and that the new constraint at each level is $\phi^{(0)}$. We can see that the constraints are first class if we use the Poisson brackets

$$
\left\{E^{(k)}, t_{(j)}\right\}=\delta_{j}^{k}, \quad\left\{x_{(k)}^{a}, p_{b}^{(j)}\right\}=\delta_{b}^{a} \delta_{k}^{j}, \quad k, j=0,1, \ldots, M .
$$

For each $M$, eliminating the $M+1$ momenta $p_{a}^{(k)}$, energies $E^{(k)}$ and multipliers $e_{(k)}$ one can recover the corresponding action in configuration space [1].

Prompted by the standard Galilean boost and rotation transformations on momenta and energy

$$
\delta p_{a}^{(0)}=m v_{(0) a}, \quad \delta E^{(0)}=v_{(0)}^{a} p_{a}^{(0)},
$$

we propose the following generalized transformations for momenta and energies

$$
\begin{aligned}
\delta p_{a}^{(k)} & =\sum_{j=0}^{M-k-1} v_{(j) a} E^{(k+j+1)}+\frac{m}{c^{2 M}} v_{(M-k) a}+\sum_{j=0}^{M-k} \omega_{(j) a b} p^{(k+j) b}, \\
\delta E^{(k)} & =\sum_{j=0}^{M-k} v_{(j)}^{a} p_{a}^{(k+j)} .
\end{aligned}
$$

It can be seen that they realize the algebra (1.6) truncated at level $M$, with central extension $H^{(M+1)}=-m / c^{2 M}$. Furthermore, as shown in appendix A, for given $M$ all the constraints $\phi^{(k)}, k=0,1, \ldots, M$, are invariant under these transformations of momenta and energies, and this provides a further justification for the form of the constraints (2.2) for general $M$. The kinetical term in the action $-E^{(k)} \dot{t}_{(k)}+p_{a}^{(k)} \dot{x}_{(k)}^{a}$ is quasi-invariant under transformations (2.12) and the corresponding transformations for $t_{(k)}, x_{(k)}^{a}$ (see [1] and appendix B).

\section{Symmetries of post-Galilean particle for $M=1$}

The action (1.5) for the second order expansion of a post-Galilean particle in $d+1$ spacetime $[1,23]$ without the total derivative $-\frac{m}{c^{2}} \dot{t}_{(2)}$ becomes

$$
S_{2}=\frac{m}{c^{2}} \int \mathrm{d} \tau\left(\frac{\dot{x}_{(0)}^{a} \dot{x}_{(1) a}}{\dot{t}_{(0)}}-\frac{\dot{t}_{(1)} \dot{x}_{(0)}^{2}}{2 \dot{t}_{(0)}^{2}}+\frac{\dot{x}_{(0)}^{4}}{8 \dot{t}_{(0)}^{3}}\right)=\int \mathrm{d} \tau L_{2} .
$$

The canonical momenta are given by

$$
\begin{aligned}
E^{(0)} & =-\frac{\partial L_{2}}{\partial \dot{t}_{(0)}}=-\frac{m}{c^{2}}\left(-\frac{\dot{x}_{(0)}^{a} \dot{x}_{(1) a}}{\dot{t}_{(0)}^{2}}+\frac{\dot{t}_{(1)} \dot{x}_{(0)}^{2}}{\dot{t}_{(0)}^{3}}-\frac{3}{8} \frac{\dot{x}_{(0)}^{4}}{\dot{t}_{(0)}^{4}}\right), \\
E^{(1)} & =-\frac{\partial L_{2}}{\partial \dot{t}_{(1)}}=\frac{m}{2 c^{2}} \frac{\dot{x}_{(0)}^{2}}{\dot{t}_{(0)}^{2}} \\
p_{a}^{(0)} & =\frac{\partial L_{2}}{\partial \dot{x}_{(0)}^{a}}=\frac{m}{c^{2}}\left(\frac{\dot{x}_{(0) a}}{\dot{t}_{(0)}}-\frac{\dot{t}_{(1)} \dot{x}_{(0) a}}{\dot{t}_{(0)}^{2}}+\frac{1}{2} \frac{\dot{x}_{(0)}^{2} \dot{x}_{(0) a}}{\dot{t}_{(0)}^{3}}\right), \\
p_{a}^{(1)} & =\frac{\partial L_{2}}{\partial \dot{x}_{(1)}^{a}}=\frac{m}{c^{2}} \frac{\dot{x}_{(0) a}}{\dot{t}_{(0)}}
\end{aligned}
$$


and they obey the primary first-class constraints

$$
\begin{aligned}
\phi^{(0)} & =p_{a}^{(0)} p^{(1) a}-\frac{1}{2} E^{(1) 2}-\frac{m}{c^{2}} E^{(0)}, \\
\phi^{(1)} & =\frac{1}{2} p^{(1) 2}-\frac{m}{c^{2}} E^{(1)},
\end{aligned}
$$

which agree with (2.2) for $M=1$. The Dirac Hamiltonian is given by

$$
\begin{aligned}
H_{D} & =e_{(0)} \phi^{(0)}+e_{(1)} \phi^{(1)} \\
& =e_{(0)}\left(p_{a}^{(0)} p^{(1) a}-\frac{1}{2} E^{(1) 2}-\frac{m}{c^{2}} E^{(0)}\right)+e_{(1)}\left(\frac{1}{2} p^{(1) 2}-\frac{m}{c^{2}} E^{(1)}\right),
\end{aligned}
$$

and yields the equations of motion

$$
\begin{aligned}
\dot{t}_{(0)} & =-\frac{\partial H_{D}}{\partial E^{(0)}}=\frac{m}{c^{2}} e_{(0)}, & \dot{t}_{(1)} & =-\frac{\partial H_{D}}{\partial E_{1}}=e_{(0)} E^{(1)}+\frac{m}{c^{2}} e_{(1)}, \\
\dot{x}_{(0)}^{a} & =\frac{\partial H_{D}}{\partial p_{a}^{(0)}}=e_{(0)} p^{(1) a}, & \dot{x}_{(1)}^{a} & =\frac{\partial H_{D}}{\partial p_{a}^{(1)}}=e_{(0)} p^{(0) a}+e_{(1)} p^{(1) a}, \\
\dot{E}^{(0)} & =\frac{\partial H_{D}}{\partial t_{(0)}}=0, & \dot{E}^{(1)} & =\frac{\partial H_{D}}{\partial t_{(1)}}=0, \\
\dot{p}_{a}^{(0)} & =-\frac{\partial H_{D}}{\partial x_{(0)}^{a}}=0, & \dot{p}_{a}^{(1)} & =-\frac{\partial H_{D}}{\partial x_{(1)}^{a}}=0 .
\end{aligned}
$$

Space-time symmetries. The canonical generator of space-time symmetries is given by

$$
G=-E^{(0)} \eta_{(0)}-E^{(1)} \eta_{(1)}+p_{a}^{(0)} \xi_{(0)}^{a}+p_{a}^{(1)} \xi_{(1)}^{a}-\delta F,
$$

with the $\eta, \xi$ and $\delta F$ are unknown functions of $t_{(0)}, t_{(1)}, x_{(0)}$ and $x_{(1)}$, so that the spacetime symmetries are obtained as $\delta t_{(0)}=\eta_{(0)}, \delta t_{(1)}=\eta_{(1)}, \delta x_{(0)}^{a}=\xi_{(0)}^{a}, \delta x_{(1)}^{a}=\xi_{(1)}^{a}$, and then $\delta L_{2}=\frac{\mathrm{d}}{\mathrm{d} \tau} \delta F$.

The equation $\dot{G}=0$ allows one to write the following Killing equations [24, 25] for the space-time symmetries of $S_{2}$,

$$
\begin{array}{rlrl}
\partial_{a}^{0} \eta_{(0)} & =0, & & \partial^{1} \eta_{(0)}=0, \\
\partial_{a}^{1} \eta_{(1)} & =0, & & \partial_{a}^{1} \eta_{(0)}=0, \\
\partial^{1} \xi_{(0)}^{a} & =0, & & \partial_{a}^{1} \xi_{(0)}^{b}=0, \\
\partial^{0} \delta F & =0, & & \partial^{1} \delta F=0, \\
\frac{m}{c^{2}} \partial^{1} \xi_{(1) a} & =\partial_{a}^{1} \delta F, & \\
\partial_{a}^{1} \xi_{(1) b}+\partial_{b}^{1} \xi_{(1) a} & =\delta_{a b} \partial^{1} \eta_{(1)}, & \\
\frac{m}{c^{2}} \partial^{0} \xi_{(0) a} & =\partial_{a}^{1} \delta F, & \\
\frac{m}{c^{2}} \partial^{0} \xi_{(1) a} & =\partial_{a}^{0} \delta F, & \\
\partial_{a}^{0} \xi_{(0) b}+\partial_{a}^{1} \xi_{(1) b} & =\delta_{a b} \partial^{0} \eta_{(0)}, & & \\
\partial_{a}^{0} \xi_{(1) b}+\partial_{a}^{0} \xi_{(1) b} & =\delta_{a b} \partial^{0} \eta_{(1)}, & &
\end{array}
$$




$$
\begin{aligned}
& \partial^{0} \eta_{(0)}=2 \partial^{1} \eta_{(1)}, \\
& \partial_{a}^{0} \eta_{(1)}=\partial^{1} \xi_{(1) a},
\end{aligned}
$$

for $a, b=1, \ldots, d$, and with $\partial^{0}=\frac{\partial}{\partial t_{(0)}}, \quad \partial^{1}=\frac{\partial}{\partial t_{(1)}}, \quad \partial_{a}^{0}=\frac{\partial}{\partial x_{(0)}^{a}}, \quad \partial_{a}^{1}=\frac{\partial}{\partial x_{(1)}^{a}}$.

These PDE can be integrated starting from the trivial ones (3.14)-(3.17), and one gets the unique solution given by

$$
\begin{aligned}
\eta_{(0)}\left(t_{(0)}\right) & =\frac{4}{3} \lambda t_{(0)}+\delta_{(0)}, \\
\eta_{(1)}\left(t_{(0)}, t_{(1)}, x_{(0)}\right)= & \frac{2}{3} \lambda t_{(1)}+v_{(0)}^{a} x_{(0) a}+\mu t_{(0)}^{2}+\lambda_{(1)} t_{(0)}+\delta_{(1)}, \\
\xi_{(0) a}\left(t_{(0)}, x_{(0)}\right)= & \lambda x_{(0) a}+v_{(0) a} t_{(0)}+\omega_{(0) a b} x_{(0)}^{b}+\varepsilon_{(0) a}, \\
\xi_{(1) a}\left(t_{(0)}, t_{(1)}, x_{(0)}, x_{(1)}\right)= & \frac{1}{3} \lambda x_{(1) a}+v_{(0) a} t_{(1)}+\mu t_{(0)} x_{(0) a}+v_{(1) a} t_{(0)} \\
& +\frac{1}{2} \lambda_{(1)} x_{(0)}+\omega_{(0) a b} x_{(1)}^{b}+\omega_{(1) a b} x_{(0)}^{b}+\varepsilon_{(1) a}, \\
\delta F\left(x_{(0)}, x_{(1)}\right)= & \frac{m}{2 c^{2}}\left(\mu x_{(0)}^{a} x_{(0) a}+2 v_{(0) a} x_{(1)}^{a}+2 v_{(1) a} x_{(0)}^{a}\right),
\end{aligned}
$$

with $\lambda, \lambda_{(1)}, \mu, \delta_{(0)}, \delta_{(1)}, \varepsilon_{(0)}^{a}, \varepsilon_{(1)}^{a}, v_{(0)}^{a}, v_{(1)}^{a}, \omega_{(0)}^{a b}$, and $\omega_{(1)}^{a b}$ arbitrary constants, where $\omega_{(0) a b}=-\omega_{(0) b a}, \omega_{(1) a b}=-\omega_{(1) b a}$.

The non-zero (or non-constant) value of $\delta F$ is associated to the fact that we dropped the total derivative $-\frac{m}{c^{2}} \dot{t}_{(2)}$ from the action $S_{(2)}$. Had we kept that term, we would have obtained $\delta t_{(2)}=-\frac{c^{2}}{m} \delta F$ (plus an arbitrary constant corresponding to shifts in $t_{(2)}$ ).

The generator of the point transformations is

$$
\begin{aligned}
G= & -E^{(0)}\left(\frac{4}{3} \lambda t_{(0)}+\delta_{(0)}\right)-E^{(1)}\left(\frac{2}{3} \lambda t_{(1)}+v_{(0)}^{a} x_{(0) a}+\mu t_{(0)}^{2}+\lambda_{(1)} t_{(0)}+\delta_{(1)}\right) \\
+ & p^{(0) a}\left(\lambda x_{(0) a}+v_{(0) a} t_{(0)}+\omega_{(0) a b} x_{(0)}^{b}+\varepsilon_{(0) a}\right) \\
+ & p^{(1) a}\left(\frac{1}{3} \lambda x_{(1) a}+v_{(0) a} t_{(1)}+\mu t_{(0)} x_{(0) a}+v_{(1) a} t_{(0)}\right. \\
& \left.\quad+\frac{1}{2} \lambda_{(1)} x_{(0)}+\omega_{(0) a b} x_{(1)}^{b}+\omega_{(1) a b} x_{(0)}^{b}+\varepsilon_{(1) a}\right) \\
& -\frac{m}{2 c^{2}}\left(\mu x_{0}^{a} x_{(0) a}+2 v_{(0) a} x_{(1)}^{a}+2 v_{(1) a} x_{(0)}^{a}\right)
\end{aligned}
$$

from which the individual generators can be defined,

$$
\begin{aligned}
& \lambda \rightarrow \quad D=-\frac{4}{3} E^{(0)} t_{(0)}-\frac{2}{3} E^{(1)} t_{(1)}+p_{a}^{(0)} x_{(0)}^{a}+\frac{1}{3} p_{a}^{(1)} x_{(1)}^{a}, \\
& \delta_{(0)} \rightarrow H^{(0)}=-E^{(0)}, \\
& \delta_{(1)} \rightarrow H^{(1)}=-E^{(1)}, \\
& \varepsilon_{(0) a} \rightarrow P^{(0)_{a}}=p_{a}^{(0)}, \\
& \varepsilon_{(1) a} \rightarrow P^{(1)_{a}}=p_{a}^{(1)}, \\
& \mu \rightarrow \quad C=-E^{(1)} t_{(0)}^{2}+t_{(0)} p_{a}^{(1)} x_{(0)}^{a}-\frac{1}{2} \frac{m}{c^{2}} x_{(0)}^{a} x_{(0) a},
\end{aligned}
$$




\begin{tabular}{|c|c|c|c|c|c|c|c|c|c|c|}
\hline$X$ & $C$ & $H^{(0)}$ & $H^{(1)}$ & $P^{(0)}$ & $P^{(1)}$ & $B^{(0)}$ & $B^{(1)}$ & $D^{(1)}$ & $J^{(0)}$ & $J^{(1)}$ \\
\hline$\Delta_{X}$ & 2 & $-\frac{4}{3}$ & $-\frac{2}{3}$ & -1 & $-\frac{1}{3}$ & $\frac{1}{3}$ & 1 & $\frac{2}{3}$ & 0 & $\frac{2}{3}$ \\
\hline
\end{tabular}

Table 1. Dilatation weights of the symmetry generators for $L_{2}$. Weights of $t_{(0)}, t_{(1)}, x_{(0)}$ and $x_{(1)}$ are the opposites of $H^{(0)}, H^{(1)}, P^{(0)}$ and $P^{(1)}$, respectively.

$$
\begin{aligned}
& \lambda_{(1)} \rightarrow D^{(1)}=-E^{(1)} t_{(0)}+\frac{1}{2} p_{a}^{(1)} x_{(0)} a \\
& v_{(0)}^{a} \rightarrow B_{a}^{(0)}=-E^{(1)} x_{(0) a}+t_{(0)} p_{a}^{(0)}+t_{(1)} p_{a}^{(1)}-\frac{m}{c^{2}} x_{(1) a}, \\
& v_{(1)}^{a} \rightarrow B_{a}^{(1)}=t_{(0)} p_{a}^{(1)}-\frac{m}{c^{2}} x_{(0) a}, \\
& \omega_{(0)}^{a b} \rightarrow J_{a b}^{(0)}=p_{a}^{(0)} x_{(0) b}-p_{b}^{(0)} x_{(0) a}+p_{a}^{(1)} x_{(1) b}-p_{b}^{(1)} x_{(1) a}, \\
& \omega_{(1)}^{a b} \rightarrow J_{a b}^{(1)}=p_{a}^{(1)} x_{(0) b}-p_{b}^{(1)} x_{(0) a} .
\end{aligned}
$$

$H^{(0)}, H^{(1)}, P^{(0)}$ and $P^{(1)}$ generate the translations in $t_{(0)}, t_{(1)}, x_{(0)}$ and $x_{(1)}$, respectively. $D$ is the generator of dilatations, $C$ is a generator of mixing-level special conformal transformations, $D^{(1)}$ generates mixing level dilatations and $B^{(0)}$ and $B^{(1)}$ are generators of mixing-level boosts. Finally, $J^{(0)}$ generates standard rotations at all levels, while $J^{(1)}$ rotates the $x_{(1)}$ but puts the result in the space of $x_{(0)}$.

Extended space-time algebra. Using the Poisson brackets (2.10) for $M=1$ one can compute the dilatation weights $\Delta_{X}$ of the generators, $\{X, D\}=\Delta_{X} X$ given in table 1 . The standard dynamical exponent, given by the quotient of the weights of $H^{(0)}$ and $P^{(0)}$, is $z=4 / 3$, in contrast to the $z=2$ value of the $M=0$ case.

The remaining non-zero brackets among generators are

$$
\begin{aligned}
\left\{H^{(0)}, C\right\} & =-2 D^{(1)}, & \left\{H^{(0)}, D^{(1)}\right\} & =-H^{(1)}, \\
\left\{H^{(0)}, B_{a}^{(1)}\right\} & =-P_{a}^{(0)}, & \left\{H^{(0)}, B_{a}^{(1)}\right\} & =-P_{a}^{(1)}, \\
\left\{H^{(1)}, B_{a}^{(0)}\right\} & =-P_{a}^{(1)}, & & \\
\left\{P_{a}^{(0)}, C\right\} & =-B_{a}^{(1)}, & \left\{P_{a}^{(0)}, D^{(1)}\right\} & =-\frac{1}{2} P_{a}^{(1)}, \quad\left\{P_{a}^{(0)}, B_{b}^{(0)}\right\}=-\delta_{a b} H^{(1)}, \\
\left\{P_{a}^{(0)}, B_{b}^{(1)}\right\} & =\frac{m}{c^{2}} \delta_{a b}, & \left\{P_{a}^{(0)}, J_{b c}^{(1)}\right\} & =\delta_{a b} P_{c}^{(1)}-\delta_{a c} P_{b}^{(1)}, \\
\left\{P_{a}^{(1)}, B_{b}^{(0)}\right\} & =\frac{m}{c^{2}} \delta_{a b}, & \left\{D^{(1)}, B_{a}^{(0)}\right\} & =-\frac{1}{2} B_{a}^{(1)}, \quad\left\{B_{a}^{(0)}, B_{b}^{(0)}\right\}=J_{a b}^{(1)}, \\
\left\{B_{a}^{(0)}, J_{b c}^{(1)}\right\} & =\delta_{a b} B_{c}^{(1)}-\delta_{a c} B_{b}^{(1)}, & &
\end{aligned}
$$

plus the rotation algebra of $J^{(0)}$ with itself and with all the generators with vector indexes. The central extension $H^{(2)}=m / c^{2}$ appears in both mixing-level translation-boost brackets $\left\{P^{(0)}, B^{(1)}\right\}$ and $\left\{P^{(1)}, B^{(0)}\right\}$, instead of in $\left\{P^{(0)}, B^{(0)}\right\}$ as in the Schrödinger algebra.

Notice that $D^{(1)}$ transforms $B$ into $B, P$ into $P$ and $H$ into $H$, but changes the level from ${ }^{(0)}$ to ${ }^{(1)}$ in each case. In this sense it acts like a higher order dilatation, and we will refer to it as a generalized dilatation. 
The generators $H^{(0)}, H^{(1)}, D, C$ and $D^{(1)}$ form a solvable, indecomposable 5dimensional subalgebra. This is in contrast with the case of the Galilean particle, where $H=H^{(0)}, C$ and $D$ are a realization of the semisimple algebra $s l(2, \mathbb{R})$.

Non-relativistic systems with higher order derivatives and with an extended phase space $x_{(n)}, p^{(n)}$ with a single dilatation and Hamiltonian have been proposed, see for example [31-34].

\section{Generalized Schrödinger algebras}

We could proceed now to the $M=2$ action and follow the same procedure as before. However, for higher $M$ the computations become very involved quite rapidly and, in particular, solving the resulting system of PDE associated to the conservation of $G$ requires the use of computer algebra packages.

Instead, in order to obtain results for arbitrary $M$, we rely on the knowledge of the $M+1$ constraints presented in section 2 and propose a generalization of the extended generators found for $M=0$ and $M=1$. Invariance of the constraints under the extended generators, together with the quasi-invariance of the kinetic terms in the canonical action, justify this approach. The form of the generators is further validated by the closure of the Poisson bracket algebra of generators.

The generators of symmetries of $L_{M+1}, M=0,1, \ldots$, that we propose are

$$
\begin{aligned}
D= & \frac{1}{2 M+1} \sum_{l=0}^{M}(2 M+1-2 l) p_{a}^{(l)} x_{(l)}^{a}-\frac{1}{2 M+1} \sum_{l=0}^{M}(2 M+2-2 l) E^{(l)} t_{(l)}, \\
D^{(k)}= & \frac{1}{2(M+1-k)} \sum_{l=k}^{M}(2(M-l)+1) p_{a}^{(l)} x_{(l-k)}^{a} \\
& -\frac{1}{M+1-k} \sum_{l=k}^{M}(M+1-l) E^{(l)} t_{(l-k)}, \\
C= & -E^{(M)} t_{(0)}^{2}+t_{(0)} p_{a}^{(M)} x_{(0)}^{a}-\frac{m}{2 c^{2 M}} x_{(0)}^{a} x_{(0) a}, \\
B_{a}^{(k)}= & \sum_{l=k}^{M} p_{a}^{(l)} t_{(l-k)}-\sum_{l=k}^{M-1} E^{(l+1)} x_{(l-k) a}-\frac{m}{c^{2 M}} x_{(M-k) a} \\
H^{(k)}= & -E^{(k)} \\
P_{a}^{(k)}= & p_{a}^{(k)} \\
J_{a b}^{(k)}= & \sum_{l=k}^{M}\left(p_{a}^{(l)} x_{(l-k) b}-x_{(l-k) a} p_{b}^{(l)}\right)
\end{aligned}
$$

where $k=0, \ldots, M$ for all the families of generators, except for the $D^{(k)}$, for which $k=$ $1, \ldots, M$. One has thus $2 M+3$ scalar generators $D, D^{(k)}, C$ and $H^{(k)}$, plus $2(M+1) d$ generators $P_{a}^{(k)}, B_{a}^{(k)}$ and $(M+1) \frac{d(d-1)}{2}$ generators $J_{a b}^{(k)}$, yielding a grand total of

$$
2 M+3+(M+1) \frac{d(d+3)}{2}
$$

generators. 
Using the Poisson brackets (2.10) one can compute the action of these generators on the canonical variables $x_{k}, p^{(k)}, t_{(k)}, E^{(k)}, k=0,1, \ldots, M$, and the results are given in appendix B. For boosts and rotations the transformations are, after multiplying by the corresponding parameters, those given in (2.12).

That the above generators correspond to symmetries of $S_{M+1}^{c}$ is proved as follows. First one can check (see appendix C) that the constraints $\phi^{(k)}, k=0,1, \ldots, M$ are invariant under the above transformations, that is,

$$
\delta^{X} \phi^{(k)}=\left\{\phi^{(k)}, X\right\}=0
$$

for $X$ in the set $\left\{D, D^{(j)}, C, H^{(j)}, P_{a}^{(j)}, B_{a}^{(j)}, J_{a b}^{(j)}\right\}$.

Furthermore, using the results in appendix B and the commutation of the transformations with the derivation with respect to $\tau$, one can prove that

$$
K=-\sum_{k=0}^{M}\left(E^{(k)} \dot{t}_{(k)}+p_{a}^{(k)} \dot{x}_{(k)}^{a}\right)
$$

is also invariant, except for the transformations corresponding to the boosts and the special conformal transformation, for which ${ }^{5}$

$$
\begin{aligned}
\delta_{a}^{B, j} K & =\frac{\mathrm{d}}{\mathrm{d} \tau}\left(\frac{m}{c^{2 M}} x_{(M-j) a}\right) \\
\delta^{C} K & =\frac{\mathrm{d}}{\mathrm{d} \tau}\left(\frac{m}{2 c^{2 M}} x_{(0)}^{a} x_{(0) a}\right) .
\end{aligned}
$$

This means that the canonical Lagrangian in $S_{M+1}^{c}$ is not invariant under the full set of transformations, but it is quasi-invariant,

$$
\delta^{G} L_{M+1}=\frac{\mathrm{d}}{\mathrm{d} \tau} \delta F_{(M)}
$$

with

$$
\delta F_{(M)}=\frac{m}{2 c^{2 M}}\left(\mu x_{(0)}^{a} x_{(0) a}+2 \sum_{k=0}^{M} v_{(k)}^{a} x_{(M-k) a},\right)
$$

where $\mu$ is the parameter of the special conformal transformation and the $v_{(k)}^{a}$ are the boosts parameters. This agrees with the known result for $M=0$ and the result for $M=1$ obtained in this paper and, as in those cases, $\delta F_{(M)}$ is associated with the dropping of the total derivative $-m / c^{2 M} \dot{t}_{(M+1)}$ in $S_{(M+1)}$.

This concludes the proof that our generators yield symmetry transformations of the canonical action. Furthermore, they form a closed algebra. Indeed, the brackets of the $D$

\footnotetext{
${ }^{5}$ We use $\delta_{a}^{B, j}$ to denote the transformation under $B_{a}^{(j)}$. This kind of notation will also be employed for the other generators later on.
} 
and $D^{(k)}$ with all the generators are

$$
\begin{aligned}
& \left\{D, D^{(k)}\right\}=-\frac{2 k}{2 M+1} D^{(k)}, \quad k=1, \ldots, M, \\
& \{D, C\}=-2 C, \\
& \left\{D, B_{a}^{(k)}\right\}=-\frac{2 k+1}{2 M+1} B_{a}^{(k)}, \quad k=0, \ldots, M, \\
& \left\{D, H^{(k)}\right\}=\frac{2 M+2-2 k}{2 M+1} H^{(k)}, \quad k=0, \ldots, M, \\
& \left\{D, P_{a}^{(k)}\right\}=\frac{2 M+1-2 k}{2 M+1} P_{a}^{(k)}, \quad k=0, \ldots, M, \\
& \left\{D, J_{a b}^{(k)}\right\}=-\frac{2 k}{2 M+1} J_{a b}^{(k)}, \quad k=0, \ldots, M, \\
& \left\{D^{(k)}, D^{(j)}\right\}=(k-j) \frac{M+1-k-j}{(M+1-k)(M+1-j)} D^{(k+j)}, \quad k, j=1, \ldots, M, \\
& \text { provided that } k+j \leq M \text {, zero otherwise, } \\
& \left\{D^{(k)}, C\right\}=0, \quad k=1, \ldots, M, \\
& \left\{D^{(k)}, B_{a}^{(j)}\right\}=-\frac{2 j+1}{2(M+1-k)} B_{a}^{(k+j)}, \quad k=1, \ldots, M, \quad j=0, \ldots, M, \\
& \text { provided that } k+j \leq M \text {, zero otherwise, } \\
& \left\{D^{(k)}, H^{(j)}\right\}=\frac{M+1-k-j}{M+1-k} H^{(k+j)}, \quad k=1, \ldots, M, \quad j=0, \ldots, M, \\
& \text { provided that } k+j \leq M \text {, zero otherwise, } \\
& \left\{D^{(k)}, P_{a}^{(j)}\right\}=\frac{2 M+1-2(k+j)}{2(M+1-k)} P_{a}^{(k+j)}, \quad k=1, \ldots, M, \quad j=0, \ldots, M, \\
& \text { provided that } k+j \leq M \text {, zero otherwise, } \\
& \left\{D^{(k)}, J_{a b}^{(j)}\right\}=-\frac{j}{M+1-k} J_{a b}^{(k+j)}, \quad k=1, \ldots, M, \quad j=0, \ldots, M, \\
& \text { provided that } k+j \leq M \text {, zero otherwise. }
\end{aligned}
$$

From (4.17) and (4.18) for $k=0$ one can see that the standard dynamical exponent associated to the Galilean variables $t_{(0)}, x_{(0)}$ depends on $M$ and is given by

$$
z_{M}=\frac{2 M+2}{2 M+1}, \quad M=0,1,2, \ldots
$$

One has that $\lim _{M \rightarrow \infty} z_{M}=1$, but (4.26) takes into account only a small part of the relations, and it is not obvious what this implies at the level of the algebra itself.

As we already noticed for $M=1$, the above relations show that $D^{(k)}$ are a higher order version of the dilatation $D$, increasing by $k$ the levels of the generators. Furthermore, if we redefine

$$
\begin{array}{ll}
L_{k} & =(M+1-k) D^{(k)}, \quad k=1, \ldots, M, \\
L_{0} & =\frac{2 M+1}{2} D,
\end{array}
$$


one has the non-negative part of a truncated Witt algebra,

$$
\left\{L_{n}, L_{m}\right\}=(n-m) L_{n+m}, \quad n, m=0, \ldots, M
$$

provided that $n+m \leq M$, and zero otherwise. The remaining brackets are

$$
\begin{aligned}
& \left\{B_{a}^{(k)}, B_{b}^{(j)}\right\}=J_{a b}^{(k+j+1)}, \quad k, j=0, \ldots, M, \\
& \text { provided that } k+j+1 \leq M \text {, zero otherwise, } \\
& \left\{J_{a b}^{(k)}, J_{c d}^{(j)}\right\}=\delta_{a d} J_{b c}^{(k+j)}+\delta_{b c} J_{a d}^{(k+j)}-\delta_{a c} J_{b d}^{(k+j)}-\delta_{b d} J_{a c}^{(k+j)}, \quad k, j=0, \ldots, M, \\
& \text { provided that } k+j \leq M \text {, zero otherwise, } \\
& \left\{B_{a}^{(k)}, J_{b c}^{(j)}\right\}=\delta_{a b} B_{c}^{(k+j)}-\delta_{a c} B_{b}^{(k+j)}, \quad k, j=0, \ldots, M, \\
& \text { provided that } k+j \leq M \text {, zero otherwise, } \\
& \left\{P_{a}^{(k)}, J_{b c}^{(j)}\right\}=\delta_{a b} P_{c}^{(k+j)}-\delta_{a c} P_{b}^{(k+j)}, \quad k, j=0, \ldots, M, \\
& \text { provided that } k+j \leq M \text {, zero otherwise, } \\
& \left\{H^{(k)}, B_{a}^{(j)}\right\}=-P_{a}^{(k+j)}, \quad k, j=0, \ldots, M, \\
& \text { provided that } k+j \leq M \text {, zero otherwise, } \\
& \left\{P_{a}^{(k)}, B_{b}^{(j)}\right\}=-\delta_{a b} H^{(k+j+1)}+\frac{m}{c^{2 M}} \delta_{a b} \delta_{M}^{k+j}, \quad k, j=0, \ldots, M, \\
& \text { provided that } k+j+1 \leq M \text {, zero otherwise, } \\
& \left\{C, B_{a}^{(k)}\right\}=0, \\
& k=0, \ldots, M \text {, } \\
& \left\{C, J_{a b}^{(k)}\right\}=0, \\
& k=0, \ldots, M \text {, } \\
& \left\{C, P_{a}^{(k)}\right\}=\delta_{0}^{k} B_{a}^{(M)}, \\
& k=0, \ldots, M \text {, }
\end{aligned}
$$

The special behaviour of the last relation for $M=0$ is what makes the $s l(2, \mathbb{R})$ algebra of $D, C, H^{(0)}$ to appear for $M=0$, while for $M>0$ the $2 M+3$ generators $C, D, D^{(k)}, H^{(k)}$ form a solvable algebra (this is because $D$ does not appear in the right-hand sides for $M>0, C$ disappears after the first derivation, and the $D^{(k)}$ and $H^{(k)}$ are dropped in successive derivations of the algebra). The central extension $H^{(M+1)}=m / c^{2 M}$ appears in brackets between translations and boosts whose indexes add to $M$.

Although some of the generators can be redefined so that some of the structure constants become independent of the level $M$, as was done for the $D, D^{(k)}$, there are some structure constants for which the dependence on $M$ cannot be erased, that is, increasing $M$ not only brings in new generators but it also changes the brackets of some of the old ones. The structure constants of the brackets among $H^{(k)}, P^{(k)}, B^{(k)}$ and $J^{(k)}$, that is, those of the original algebra (1.6), as well as those of $C$ with $P^{(k)}, B^{(k)}$ and $J^{(k)}$, do not depend on $M$ (the $1 / c^{2 m}$ can be absorbed into $m$ ). Using the $L_{n}$ instead of $D, D^{(k)}$, the 
remaining brackets, besides (4.29), are

$$
\begin{aligned}
\left\{L_{n}, B_{a}^{(k)}\right\} & =-\frac{2 k+1}{2} B_{a}^{(k+n)} \\
\left\{L_{n}, P_{a}^{(k)}\right\} & =\frac{2 M+1-2(n+k)}{2} P_{a}^{(k+n)} \\
\left\{L_{n}, J_{a b}^{(k)}\right\} & =-k J_{a b}^{(k+n)} \\
\left\{L_{n}, H^{(k)}\right\} & =(M+1-(n+k)) H^{(k+n)} \\
\left\{L_{n}, C\right\} & =-\delta_{n 0}(2 M+1) C \\
\left\{C, H^{(k)}\right\} & =2 \delta_{0}^{k} L_{M}
\end{aligned}
$$

From this is clear that, for instance, one cannot redefine $P^{(k)}$ so that the dependence on $M$ dissapears from (4.41), and the same happens for (4.43) and (4.44), while (4.45) has the problem that the generator in the right-hand side depends itself on $M$.

\section{Generalized Schrödinger equation and projective invariance}

The quantization of the systems that we have considered can be performed by imposing the canonical constraints on the physical states of the corresponding Hilbert space. For $M=1$ we have two constraints and we obtain a set of two generalized Schrödinger equations,

$$
\begin{aligned}
-\frac{\partial^{2} \Psi}{\partial x_{(0) a} \partial x_{(1)}^{a}}+\frac{1}{2} \frac{\partial^{2} \Psi}{\partial t_{(1)}^{2}}-i \frac{m}{c^{2}} \frac{\partial \Psi}{\partial t_{(0)}} & =0, \\
-\frac{1}{2} \frac{\partial^{2} \Psi}{\partial x_{(1) a} \partial x_{(1)}^{a}}-i \frac{m}{c^{2}} \frac{\partial \Psi}{\partial t_{(1)}} & =0
\end{aligned}
$$

where $\Psi\left(t_{(0)}, t_{(1)}, x_{(0)}, x_{(1)}\right)$ is the wave function of the physical state in coordinate representation. Working in $d=1$ for simplicity and looking for solutions of the form

$$
\Psi\left(t_{(0)}, t_{(1)}, x_{(0)}, x_{(1)}\right)=\Psi_{0}\left(t_{(0)}, x_{(0)}\right) \Psi_{1}\left(t_{(1)}, x_{(1)}\right),
$$

equation (5.2) can be solved by separation of variables with separation constant $\varepsilon$ to obtain

$$
\begin{aligned}
\Psi_{1}\left(t_{(1)}, x_{(1)}\right) & =A_{+} e^{-i\left(\varepsilon t_{(1)}-\frac{\sqrt{2 m \varepsilon}}{c} x_{(1)}\right)}+A_{-} e^{-i\left(\varepsilon t_{(1)}+\frac{\sqrt{2 m \varepsilon}}{c} x_{(1)}\right)} \\
& \equiv \Psi_{+}\left(t_{(1)}, x_{(1)}\right)+\Psi_{-}\left(t_{(1)}, x_{(1)}\right)
\end{aligned}
$$

where $A_{ \pm}$are arbitrary constants.

The separation constant $\varepsilon$ characterizes the dependence of the wave function on $t_{(1)}$, $x_{(1)}$, and is the common eigenvalue of the operators corresponding to $E^{(1)}$ and $\frac{c^{2}}{2 m} P^{(1) 2}$, that is, $i \partial_{t(1)}$ and $-\frac{c^{2}}{2 m} \partial_{x(1)}^{2}$, respectively.

Each of the $\Psi_{ \pm}\left(t_{(1)}, x_{(1)}\right)$ can be substituted into (5.1) and one obtains a first-order $\operatorname{PDE}$ for $\Psi_{0}$,

$$
-i\left( \pm \frac{\sqrt{2 m \varepsilon}}{c}\right) \frac{\partial \Psi_{0}}{\partial x_{(0)}}-\frac{1}{2} \varepsilon^{2} \Psi_{0}-i \frac{m}{c^{2}} \frac{\partial \Psi_{0}}{\partial t_{(0)}}=0
$$


Equation (5.4) can be solved by the method of characteristics. Imposing the initial condition $\Psi_{0}\left(0, x_{(0)}\right)=F\left(x_{(0)}\right)$ at $t_{(0)}=0$, with $F$ an arbitrary smooth function, one obtains,

$$
\Psi_{0}\left(t_{(0)}, x_{(0)}\right)=F_{ \pm}\left(x_{(0)}-\frac{c}{m}( \pm \sqrt{2 m \varepsilon}) t_{(0)}\right) e^{i \frac{c^{2}}{2 m} \varepsilon^{2} t_{(0)}}
$$

where $F_{+}$and $F_{-}$are the arbitrary functions corresponding to the \pm signs in (5.4). Finally, the total wave function solution to the system of Schrödinger equations is

$$
\begin{aligned}
\Psi_{\varepsilon}\left(t_{(0)}, t_{(1)}, x_{(0)}, x_{(1)}\right)= & F_{+}\left(x_{(0)}-\frac{c}{m} \sqrt{2 m \varepsilon} t_{(0)}\right) e^{-i\left(\varepsilon t_{(1)}-\frac{\sqrt{2 m \varepsilon}}{c} x_{(1)}-\frac{c^{2}}{2 m} \varepsilon^{2} t_{(0)}\right)} \\
& +F_{-}\left(x_{(0)}+\frac{c}{m} \sqrt{2 m \varepsilon} t_{(0)}\right) e^{-i\left(\varepsilon t_{(1)}+\frac{\sqrt{2 m \varepsilon}}{c} x_{(1)}-\frac{c^{2}}{2 m} \varepsilon^{2} t_{(0)}\right)},
\end{aligned}
$$

where we have written a $\varepsilon$ sub-index to indicate the dependence on the parameter $\varepsilon$. Alternatively, we can identify the solution using $p$ to refer to the two eigenvalues $\pm p$ of the momentum operator $-i \partial_{x_{(1)}}$ for the two components, with $\varepsilon=c^{2} /(2 m) p^{2}$, and write

$$
\begin{aligned}
\Psi_{p}\left(t_{(0)}, t_{(1)}, x_{(0)}, x_{(1)}\right)= & F_{+}\left(x_{(0)}-\frac{c^{2}}{m} p t_{(0)}\right) e^{-i\left(\frac{c^{2}}{2 m} p^{2} t_{(1)}-p x_{(1)}-\frac{c^{6}}{8 m^{3}} p^{4} t_{(0)}\right)} \\
& +F_{-}\left(x_{(0)}+\frac{c^{2}}{m} p t_{(0)}\right) e^{-i\left(\frac{c^{2}}{2 m} p^{2} t_{(1)}+p x_{(1)}-\frac{c^{6}}{8 m^{3}} p^{4} t_{(0)}\right)}
\end{aligned}
$$

This method can be repeated to solve the system of Schrödinger equations for any $M>1$. The constraint for $k=M$ yields a equation for the dependence on $t_{(M)}, x_{(M)}$ which is second order in $x_{(M)}$ and which can be solved by separation of variables, yielding left and right travelling waves in $t_{(M)}, x_{(M)}$. Each of the two solutions can then be substituted in the equation for $k=M-1$, and one gets a first order PDE in $t_{(M-1)}, x_{(M-1)}$, which can be solved by the method of characteristics. The obtained solutions can be substituted in the equation for $k=M-2$ which is again of first order in $t_{(M-2)}, x_{(M-2)}$, and the procedure can be iterated until we reach the equation for $k=0$.

Projective phase. We will discuss here the transformation properties of the above wave functions and Schrödinger equations and the associated projective phases under the postGalilean transformations. For simplicity we will work again in $d=1$. We will discuss the case of expansions in detail and summarize the results for the other transformations. Since we have $d=1$ we do not consider rotations and generalized rotations. Also, it should be taken into account that the finite transformations presented below are those of the individual generators.

1. Expansions $\boldsymbol{C}$. The expansions for $M=1$ define a vector field in the cotangent manifold, with coordinates $\left(t_{(0)}, x_{(0)}, t_{(1)}, x_{(1)}, E^{(0)}, E^{(1)}, p^{(0)}, p^{(1)}\right)$, given by

$$
\begin{aligned}
X^{C}= & t_{(0)} x_{(0)} \frac{\partial}{\partial x_{(1)}}+t_{(0)}^{2} \frac{\partial}{\partial t_{(1)}}+\left(-t_{(0)} p^{(1)}+\frac{m}{c^{2}} x_{(0)}\right) \frac{\partial}{\partial p^{(0)}} \\
& +\left(-2 E^{(1)} t_{(0)}+p^{(1)} x_{(0)}\right) \frac{\partial}{\partial E^{(0)}}
\end{aligned}
$$


which, after a trivial integration, yields the finite expansions

$$
\begin{array}{ll}
\hat{x}_{(0)}=x_{(0)}, & \hat{p}^{(0)}=p^{(0)}+\mu\left(-t_{(0)} p^{(1)}+\frac{m}{c^{2}} x_{(0)}\right), \\
\hat{x}_{(1)}=x_{(1)}+\mu t_{(0)} x_{(0)}, & \hat{p}^{(1)}=p^{(1)}, \\
\hat{t}_{(0)}=t_{(0)}, & \hat{E}^{(0)}=E^{(0)}+\mu\left(-2 E^{(1)} t_{(0)}+p^{(1)} x_{(0)}\right), \\
\hat{t}_{(1)}=t_{(1)}+\mu t_{(0)}^{2}, & \hat{E}^{(1)}=E^{(1)} .
\end{array}
$$

Notice that $t_{(0)}$ and $x_{(0)}$ do not transform under $C$, while for the standard Schrödinger expansions $(M=0)$ one has

$$
\hat{t}_{(0)}=\frac{t_{(0)}}{1-\mu t_{(0)}}, \quad \hat{x}_{(0)}=\frac{x_{(0)}}{1-\mu t_{(0)}} .
$$

Let $\Psi$ be a solution to (5.1) and (5.2), and let $\hat{\Psi}$ be the transformed solution under a finite $C$ transformation. Assume that $\hat{\Psi}$ and $\Psi$ are related by

$$
\hat{\Psi}\left(\hat{t}_{(0)}, \hat{t}_{(1)}, \hat{x}_{(0)}, \hat{x}_{(1)}\right)=e^{i \varphi\left(t_{(0)}, t_{(1)}, x_{(0)}, x_{(1)}\right)} \Psi\left(t_{(0)}, t_{(1)}, x_{(0)}, x_{(1)}\right) .
$$

If we demand $\hat{\Psi}$ to satisfy (5.1) and (5.2) in the transformed coordinates one gets, after some algebra, the following set of PDE for $\varphi$

$$
\frac{\partial \varphi}{\partial t_{(1)}}=\frac{\partial \varphi}{\partial x_{(1)}}=\frac{\partial \varphi}{\partial t_{(0)}}=0, \quad \frac{\partial \varphi}{\partial x_{(0)}}-\mu \frac{m}{c^{2}} x_{(0)}=0
$$

whose solution is

$$
\varphi\left(t_{(0)}, t_{(1)}, x_{(0)}, x_{(1)}\right)=\mu \frac{m}{2 c^{2}} x_{(0)}^{2}+\text { constant. }
$$

Since the Jacobian

$$
\frac{\partial\left(\hat{x}_{(1)}, \hat{x}_{(0)}\right)}{\partial\left(x_{(1)}, x_{(0)}\right)}=\left|\begin{array}{cc}
1 & \mu t_{(0)} \\
0 & 1
\end{array}\right|=1
$$

is trivial, an imaginary part for the constant is not needed to compensate for a change in the measure in $x_{(0)}, x_{(1)}$ space, and we can take it equal to zero. Again, this is different from what happens in the $M=0$ case, for which the projective phase acquires an imaginary part,

$$
\varphi\left(t_{(0)}, x_{(0)}\right)=\frac{1}{2} \frac{m \mu}{1-\mu t_{(0)}} x_{(0)}^{2}-\frac{i}{2} \log \left|1-\mu t_{(0)}\right| .
$$

One has then

$$
|\hat{\Psi}|^{2}=\left|1-\mu t_{(0)}\right||\Psi|^{2},
$$

so that $|\hat{\Psi}|^{2} \mathrm{~d} \hat{x}_{(0)}=|\Psi|^{2} \mathrm{~d} x_{(0)}$, as desired.

Returning to the $M=1$ expansions, we have

$$
\hat{\Psi}\left(\hat{t}_{(0)}, \hat{t}_{(1)}, \hat{x}_{(0)}, \hat{x}_{(1)}\right)=e^{i \mu \frac{m}{2 c^{2}} x_{(0)}^{2}} \Psi\left(t_{(0)}, t_{(1)}, x_{(0)}, x_{(1)}\right) .
$$


Notice that the projective phase can also be obtained by iteration of the infinitesimal $\delta F$ corresponding to expansions,

$$
\varphi=\sum_{n=1}^{\infty} \frac{1}{n !} \delta^{n} F
$$

with $\delta^{n} F=\delta\left(\delta^{n-1} F\right)$. Since $\delta F=m /\left(2 c^{2}\right) \mu x_{(0)}^{2}$ and $\delta x_{(0)}=0$ for expansions in the $M=1$ case, the contributions are null after the lineal one. For a general discussion about projective phase, central extensions and invariance up to a total derivative of the Lagrangian see [35-37].

As a check, let us assume that $\Psi$ is a solution of (5.1), (5.2) given by (5.6), and let us prove that then $\hat{\Psi}$ has the same form. Using (5.6), the right-hand side of (5.20) is

$$
\begin{aligned}
& F_{+}\left(x_{(0)}-\frac{c}{m} \sqrt{2 m \varepsilon} t_{(0)}\right) e^{-i\left(\varepsilon t_{(1)}-\frac{\sqrt{2 m \varepsilon}}{c} x_{(1)}-\frac{c^{2}}{2 m} \varepsilon^{2} t_{(0)}\right)} e^{i \mu \frac{m}{2 c^{2}} x_{(0)}^{2}} \\
& +F_{-}\left(x_{(0)}+\frac{c}{m} \sqrt{2 m \varepsilon} t_{(0)}\right) e^{-i\left(\varepsilon t_{(1)}+\frac{\sqrt{2 m \varepsilon}}{c} x_{(1)}-\frac{c^{2}}{2 m} \varepsilon^{2} t_{(0)}\right)} e^{i \mu \frac{m}{2 c^{2}} x_{(0)}^{2}}, \\
& =F_{+}\left(\hat{x}_{(0)}-\frac{c}{m} \sqrt{2 m \varepsilon} \hat{t}_{(0)}\right) e^{-i\left(\varepsilon t_{(1)}-\frac{\sqrt{2 m \varepsilon}}{c} x_{(1)}-\frac{c^{2}}{2 m} \varepsilon^{2} \hat{t}_{(0)}\right)} e^{i \mu \frac{m}{2 c^{2}} \hat{x}_{(0)}^{2}} \\
& \quad+F_{-}\left(\hat{x}_{(0)}+\frac{c}{m} \sqrt{2 m \varepsilon} \hat{t}_{(0)}\right) e^{-i\left(\varepsilon t_{(1)}+\frac{\sqrt{2 m \varepsilon}}{c} x_{(1)}-\frac{c^{2}}{2 m} \varepsilon^{2} \hat{t}_{(0)}\right)} e^{i \mu \frac{m}{2 c^{2}} \hat{x}_{(0)}^{2}},
\end{aligned}
$$

where we have used that $\hat{t}_{(0)}=t_{(0)}, \hat{x}_{(0)}=x_{(0)}$. Expressing now $t_{(1)}$ and $x_{(1)}$ in terms of the transformed variables one gets

$$
\varepsilon t_{(1)} \mp \frac{\sqrt{2 m \varepsilon}}{c} x_{(1)}=\varepsilon \hat{t}_{(1)} \mp \frac{\sqrt{2 m \varepsilon}}{c} \hat{x}_{(1)}-\mu\left(\varepsilon \hat{t}_{(0)}^{2} \mp \frac{\sqrt{2 m \varepsilon}}{c} \hat{t}_{(0)} \hat{x}_{(0)}\right) .
$$

The extra terms can be combined with the one with $\hat{x}_{(0)}^{2}$ and one gets the perfect square

$$
-i \mu \frac{m}{2 c^{2}}\left(\hat{x}_{(0)} \mp \frac{c}{m} \sqrt{2 m \varepsilon} \hat{t}_{(0)}\right)^{2}
$$

whose exponential can then be absorbed into $F_{ \pm}$to yield the corresponding $\hat{F}_{ \pm}$for $\hat{\Psi}$, giving it the same form as in (5.6) but in transformed variables. Notice that the parameter $\varepsilon$ does not change when going to the transformed coordinates, since for a given solution it corresponds to the value of $E^{(1)}$, and under the expansions one has $\hat{E}^{(1)}=E^{(1)}$. This is not, however, true of some of the other transformations and the change of $E^{(1)}$, or of $\frac{c^{2}}{2 m} p^{(1) 2}$, must be taken into account. 
2. Boosts $\boldsymbol{B}^{(\mathbf{0})}$. The finite transformations for parameter $v_{(0)}=v$ are in this case

$$
\begin{aligned}
& \hat{x}_{(0)}=x_{(0)}+v t_{(0)}, \\
& \hat{p}^{(0)}=p^{(0)}+v E^{(1)}+\frac{1}{2} v^{2} p^{(1)}+\frac{1}{6} \frac{m}{c^{2}} v^{3}, \\
& \hat{x}_{(1)}=x_{(1)}+v t_{(1)}+\frac{1}{2} v^{2} x_{(0)}+\frac{1}{6} v^{3} t_{(0)}, \\
& \hat{p}^{(1)}=p^{(1)}+\frac{m}{c^{2}} v, \\
& \hat{t}_{(0)}=t_{(0)}, \\
& \hat{E}^{(0)}=E^{(0)}+v p^{(0)}+\frac{1}{2} v^{2} E^{(1)}+\frac{1}{6} v^{3} p^{(1)}+\frac{1}{24} \frac{m}{c^{2}} v^{4}, \\
& \hat{t}_{(1)}=t_{(1)}+v x_{(0)}+\frac{1}{2} v^{2} t_{(0)}, \\
& \hat{E}^{(1)}=E^{(1)}+v p^{(1)}+\frac{1}{2} \frac{m}{c^{2}} v^{2},
\end{aligned}
$$

and the projective phase is

$$
\varphi=\frac{m}{c^{2}}\left(v x_{(1)}+\frac{1}{2} v^{2} t_{(1)}+\frac{1}{6} v^{3} x_{(0)}+\frac{1}{24} v^{4} t_{(0)}\right) .
$$

3. Generalized boosts $\boldsymbol{B}^{(\mathbf{1})}$. The finite transformations corresponding to parameter $v_{(1)}=v$ are

$$
\begin{array}{ll}
\hat{x}_{(0)}=x_{(0)}, & \hat{p}^{(0)}=p^{(0)}+\frac{m}{c^{2}} v, \\
\hat{x}_{(1)}=x_{(1)}+v t_{(0)}, & \hat{p}^{(1)}=p^{(1)}, \\
\hat{t}_{(0)}=t_{(0)}, & \hat{E}^{(0)}=E^{(0)}+v p^{(1)}, \\
\hat{t}_{(1)}=t_{(1)}, & \hat{E}^{(1)}=E^{(1)} .
\end{array}
$$

The projective phase is in this case

$$
\varphi=\frac{m}{c^{2}} v x_{(0)}
$$

4. Dilatations $\boldsymbol{D}$. The finite transformations for parameter $\lambda$ are

$$
\begin{array}{ll}
\hat{x}_{(0)}=e^{\lambda} x_{(0)}, & \hat{p}^{(0)}=e^{-\lambda} p^{(0)}, \\
\hat{x}_{(1)}=e^{\lambda / 3} x_{(1)}, & \hat{p}^{(1)}=e^{-\lambda / 3} p^{(1)}, \\
\hat{t}_{(0)}=e^{4 \lambda / 3} t_{(0)}, & \hat{E}^{(0)}=e^{-4 \lambda / 3} E^{(0)}, \\
\hat{t}_{(1)}=e^{2 \lambda / 3} t_{(1)}, & \hat{E}^{(1)}=e^{-2 \lambda / 3} t_{(1)} E^{(1)} .
\end{array}
$$

The projective phase is a constant which, however, cannot be taken as zero and in fact must be imaginary $\varphi=i \frac{2}{3} \lambda$, to compensate for the change in the measure in $x_{(0)}, x_{(1)}$ space, $\mathrm{d} \hat{x}_{(0)} \mathrm{d} \hat{x}_{(1)}=e^{4 \lambda / 3} \mathrm{~d} x_{(0)} \mathrm{d} x_{(1)}$. 
5. Generalized dilatations $\boldsymbol{D}^{(\mathbf{1})}$. The finite transformations corresponding to parameter $\lambda_{(1)}=\lambda$ are

$$
\begin{array}{ll}
\hat{x}_{(0)}=x_{(0)}, & \hat{p}^{(0)}=p^{(0)}-\frac{1}{2} \lambda p^{(1)}, \\
\hat{x}_{(1)}=x_{(1)}+\frac{1}{2} \lambda x_{(0)}, & \hat{p}^{(1)}=p^{(1)}, \\
\hat{t}_{(0)}=t_{(0)}, & \hat{E}^{(0)}=E^{(0)}-\lambda E^{(1)}, \\
\hat{t}_{(1)}=t_{(1)}+\lambda t_{(0)}, & \hat{E}^{(1)}=E^{(1)} .
\end{array}
$$

and the projective phase is trivial. No constant imaginary part for $\varphi$ is needed, since $\mathrm{d} \hat{x}_{(0)} \mathrm{d} \hat{x}_{(1)}=\mathrm{d} x_{(0)} \mathrm{d} x_{(1)}$ in this case.

6. Time shifts and space translations $H^{(0)}, H^{(1)}, P^{(0)}, P^{(1)}$. The finite transformations are just shifts in the corresponding variables, the momenta do not transform and the projective phase can be chosen as zero in all the cases.

Schrödinger equation with higher derivatives. The way that we have constructed the Schrödinger equation of our system corresponds to what is known as weak quantization, where the constraints are imposed as operators on the states of the system. One can also consider a reduced space quantization, in which the gauge invariance is broken and a Hamiltonian in then computed. We can do this for our action (1.5), disregarding the total derivative, by imposing the two gauge conditions [1]

$$
t_{(0)}=c^{-2} t_{(1)}=\tau,
$$

whereby one obtains the gauge fixed action

$$
S_{(2)}^{*}=\frac{m}{c^{2}} \int \mathrm{d} t\left(\dot{x}_{(0)}^{a} \dot{x}_{(1) a}-\frac{c^{2}}{2} \dot{x}_{(0)}^{a} \dot{x}_{(0) a}+\frac{1}{8}\left(\dot{x}_{(0)}^{a} \dot{x}_{(0) a}\right)^{2}\right) .
$$

From this one can compute the Hamiltonian

$$
H_{(2)}^{*}=\frac{c^{2}}{m} p_{(0) a} p_{(1)}^{a}+\frac{1}{2} \frac{c^{4}}{m} p_{(1) a} p_{(1)}^{a}-\frac{1}{8} \frac{c^{6}}{m^{3}}\left(p_{(1) a} p_{(1)}^{a}\right)^{2},
$$

and write down the corresponding time-dependent Schrödinger equation for $\Psi\left(t, x_{(0)}, x_{(1)}\right)$

$$
i \frac{\partial \Psi}{\partial t}=-\frac{c^{2}}{m} \frac{\partial^{2} \Psi}{\partial x_{(0) a} \partial x_{(1)}^{a}}-\frac{1}{2} \frac{c^{4}}{m} \frac{\partial^{2} \Psi}{\partial x_{(1) a} \partial x_{(1)}^{a}}-\frac{1}{8} \frac{c^{6}}{m^{3}} \frac{\partial^{4} \Psi}{\partial x_{(1) a}{ }^{2} \partial x_{(1)}^{a}{ }^{2}},
$$

which is of fourth order. We can also obtain an equation of this order by plugging (5.2) into (5.1),

$$
i \frac{\partial \Psi}{\partial t_{(0)}}=-\frac{c^{2}}{m} \frac{\partial^{2} \Psi}{\partial x_{(0) a} \partial x_{(1)}^{a}}-\frac{1}{8} \frac{c^{6}}{m^{3}} \frac{\partial^{4} \Psi}{\partial x_{(1) a}^{2} \partial x_{(1)}^{a}{ }^{2}},
$$

which is also of fourth order and with the same coefficients, but lacks the term with second derivatives in $x_{(1)}$. One can conclude then that the system described by action (1.5) is one for which the two quantization procedures yield different results. Nevertheless, one should notice that the wave function obtained by using the gauge fixing (5.47) in (5.7), which by construction is a solution of (5.51) with $t_{(0)}=t$, is also a solution of (5.50). 


\section{Conclusions and outlook}

In this paper we have constructed the most general point symmetry transformations of the post Galilean actions [1] that can be obtained from the Minkowskian action for a massive particle. The algebras obtained are generalizations of the ordinary Schrödinger algebra $[15,16]$. Besides the generalized Galilean transformations, they contain dilatations, $D$, generalized dilatations $D^{(k)}$ and expansions $C$. The algebras are different from extensions of Galilean conformal algebras with dynamical exponent $z=2 / N$, with $N$ positive integer, since these contain an $\operatorname{sl}(2, \mathbb{R})$ subalgebra $[27,28]$.

Using a weak quantization procedure, we have introduced an Schrödinger equation for the post-Galilean particle that consists of $M+1$ partial differential equations, up to second order in derivatives, for a wave function living in a generalized space. Like the case of ordinary Schrödinger equation, the wave function supports a ray representation of the symmetry group, and we have calculated the projective phase for each transformation. The symmetries of generalized Schrödinger equations in this paper are different from the symmetries of the higher order Schrödinger equations [34].

If we consider the reduced space quantization the corresponding Schrödinger equation is a single differential equation of fourth order. The two procedures of quantization do not coincide in general. Further investigation of the difference between the Schrödinger equations obtained from the weak and reduced space quantizations, and the generalization of this fact for the actions $S_{(M+1)}$, will also be the subject of future work.

It will be interesting to study the relation of the higher order Schrödinger equation with the expansion up to order $v^{2} / c^{2}$ of the square root of the Klein-Gordon equation, see for example [38, 39],

$$
i \frac{\partial \Psi}{\partial t}=\sqrt{-c^{2} \frac{\partial^{2}}{\partial x^{a} \partial x_{a}}+m^{2} c^{4}} \Psi,
$$

and if it is possible to introduce interaction terms in the new Schrödinger equation that we have found.

Finally, another topic of interest for future research is the computation of quadratic invariants under stability subgroups of the generalized Schrödinger algebras and their use to construct associated space-times, as done for instance in [40] to obtain pp-wave metrics from Newton-Hooke algebras.

\section{Acknowledgments}

We would like to thank Eric Bergshoeff, Gary Gibbons, Axel Kleinschmidt, Patricio Salgado-Rebolledo and Paul Townsend for their comments on the paper. We would also like to thank the anonymous reviewer of the first version of this paper for his/her insightful observations.

The work of CB has been partially supported by Generalitat de Catalunya through project 2017 SGR 872 and by Spanish national project DOVELAR-IRI, RTI2018-096001-BC32. JG has been supported in part by MINECO FPA2016-76005-C2-1-P and Consolider 
CPAN, and by the Spanish government (MINECO/FEDER) under project MDM-20140369 of ICCUB (Unidad de Excelencia María de Maeztu).

\section{A Invariance of the contraints under generalized boosts and rotations}

Under the boosts in (2.12) the variation of the constraints (2.2) is

$$
\begin{aligned}
\delta \phi^{(k)}= & -\frac{m}{c^{2 M}} \sum_{j=0}^{M-k} v_{(j)}^{a} p_{a}^{(k+j)} \\
& +\frac{1}{2} \sum_{l=k}^{M-1} \sum_{j=0}^{M-l-1} v_{(j)}^{a} E^{(l+j+1)} p_{a}^{(M+k-l)} \\
& +\frac{1}{2} \frac{m}{c^{2 M}} \sum_{l=k}^{M} v_{(M-l)}^{a} p_{a}^{(M+k-l)} \\
& +\frac{1}{2} \sum_{l=k+1}^{M} \sum_{j=0}^{l-k-1} v_{(j)}^{a} p_{a}^{(l)} E^{(M+k-l+j+1)} \\
& +\frac{1}{2} \frac{m}{c^{2 M}} \sum_{l=k}^{M} v_{(l-k)}^{a} p_{a}^{(l)} \\
& -\frac{1}{2} \sum_{l=k}^{M-1} \sum_{j=0}^{M-l-1} v_{(j)}^{a} p_{a}^{(l+j+1)} E^{(M+k-l)} \\
& -\frac{1}{2} \sum_{l=k}^{M-1} \sum_{j=0}^{l-k} v_{(j)}^{a} E^{(l+1)} p_{a}^{(M+k-l+j)} .
\end{aligned}
$$

Sums in (A.3) and (A.5) can be brought to the same form as in (A.1) under the changes of indexes $l \rightarrow j=M-l$ and $l \rightarrow j=l-k$, respectively, and hence cancel overall. Performing the double change of indexes $(l, j) \rightarrow(\hat{l}, \hat{j})=(l-j, j)$ in (A.7) brings it to the same form as in (A.2), and hence both terms cancel each other. Finally, after performing the change $(l, j) \rightarrow(\hat{l}, \hat{j})=(l-j-1, j)$ in (A.4) one sees that it exactly cancels (A.6), and hence the constraint is invariant under boosts.

For the rotations, for which $\delta E^{(k)}=0$, one gets

$$
\delta \phi^{(k)}=\frac{1}{2} \sum_{l=k}^{M} \sum_{j=0}^{M-l} \omega_{(j) a b} p^{(l+j) b} p^{(M+k-l) a}+\frac{1}{2} \sum_{l=k}^{M} \sum_{j=0}^{l-k} \omega_{(j) a b} p^{(l) a} p^{(M+k-l+j) b} .
$$

Performing the change of indexes $(l, j) \rightarrow(\hat{l}, \hat{j})=(l-j, j)$ in the second double sum and using the skew-symmetry of the $\omega^{(k)}$ one sees that this cancels the first double sum, and hence $\delta \phi^{(k)}=0$ under rotations as well.

\section{B Transformation of the canonical variables under the $M$ th level sym- metry generators}

Given $M \geq 0$ we compute the transformation of the canonical variables $x_{(j)}, p^{(j)}, t_{(j)}$ and $E^{(j)}$ for $j=0,1, \ldots, M$. In all the following results it should be understood that if 
a canonical variable appears with an index outside of the range $[0, M]$ the corresponding result should be treated as zero.

\section{Dilatation $D$.}

$$
\begin{gathered}
\delta^{D} x_{(j)}^{a}=\left\{x_{(j)}^{a}, D\right\}=\frac{2 M+1-2 j}{2 M+1} x_{(j)}^{a}, \\
\delta^{D} p_{a}^{(j)}=\left\{p_{a}^{(j)}, D\right\}=-\frac{2 M+1-2 j}{2 M+1} p_{a}^{(j)}, \\
\delta^{D} t_{(j)}=\left\{t_{(j)}, D\right\}=\frac{2 M+2-2 j}{2 M+1} t_{(j)}, \\
\delta^{D} E^{(j)}=\left\{E^{(j)}, D\right\}=-\frac{2 M+2-2 j}{2 M+1} E^{(j)} .
\end{gathered}
$$

Generalized dilatations $D^{(k)}, k=1, \ldots, M$.

$$
\begin{aligned}
& \delta^{D, k} x_{(j)}^{a}=\left\{x_{(j)}^{a}, D^{(k)}\right\}=\frac{2(M-j)+1}{2(M+1-k)} x_{(j-k)}^{a}, \\
& \delta^{D, k} p_{a}^{(j)}=\left\{p_{a}^{(j)}, D^{(k)}\right\}=-\frac{2(M-k-j)+1}{2(M+1-k)} p_{a}^{(j+k)} \\
& \delta^{D, k} t_{(j)}=\left\{t_{(j)}, D^{(k)}\right\}=\frac{M+1-j}{M+1-k} t_{(j-k)} \\
& \delta^{D, k} E^{(j)}=\left\{E^{(j)}, D^{(k)}\right\}=-\frac{M+1-k-j}{M+1-k} E^{(j+k)}
\end{aligned}
$$

Expansions $C$.

$$
\begin{aligned}
\delta^{C} x_{(j)}^{a} & =\left\{x_{(j)}^{a}, C\right\}=\delta_{j}^{M} t_{(0)} x_{(0)}^{a}, \\
\delta^{C} p_{a}^{(j)} & =\left\{p_{a}^{(j)}, C\right\}=\delta_{0}^{j}\left(-t_{(0)} p_{a}^{(M)}+\frac{m}{c^{2 M}} x_{(0) a}\right), \\
\delta^{C} t_{(j)} & =\left\{t_{(j)}, C\right\}=\delta_{j}^{M} t_{(0)}^{2}, \\
\delta^{C} E^{(j)} & =\left\{E^{(j)}, C\right\}=\delta_{0}^{j}\left(-2 E^{(M)} t_{(0)}+p_{b}^{(M)} x_{(0)}^{b}\right) .
\end{aligned}
$$

Boosts and generalized boosts $B^{(k)}, k=0, \ldots, M$.

$$
\begin{aligned}
\delta_{a}^{B, k} x_{(j)}^{b} & =\left\{x_{(j)}^{b}, B_{a}^{(k)}\right\}=\delta_{a}^{b} t_{(j-k)}, \\
\delta_{a}^{B, k} p_{b}^{(j)} & =\left\{p_{b}^{(j)}, B_{a}^{(k)}\right\}=\delta_{a b} E^{(j+k+1)}+\frac{m}{c^{2 M}} \delta_{a b} \delta_{M-k}^{j}, \\
\delta_{a}^{B, k} t_{(j)} & =\left\{t_{(j)}, B_{a}^{(k)}\right\}=x_{(j-k-1) a}, \\
\delta_{a}^{B, k} E^{(j)} & =\left\{E^{(j)}, B_{a}^{(k)}\right\}=p_{a}^{(j+k)} .
\end{aligned}
$$

Time shifts $H^{(k)}, k=0, \ldots, M$.

$$
\begin{aligned}
\delta^{H, k} x_{(j)}^{a} & =\left\{x_{(j)}^{a}, H^{(k)}\right\}=0, \\
\delta^{H, k} p_{a}^{(j)} & =\left\{p_{a}^{(j)}, H^{(k)}\right\}=0, \\
\delta^{H, k} t_{(j)} & =\left\{t_{(j)}, H^{(k)}\right\}=\delta_{j}^{k}, \\
\delta^{H, k} E^{(j)} & =\left\{E^{(j)}, H^{(k)}\right\}=0 .
\end{aligned}
$$


Space translations $P^{(k)}, k=0, \ldots, M$.

$$
\begin{aligned}
\delta_{a}^{P, k} x_{(j)}^{b} & =\left\{x_{(j)}^{b}, P_{a}^{(k)}\right\}=\delta_{j}^{k} \delta_{a}^{b}, \\
\delta_{a}^{P, k} p_{b}^{(j)} & =\left\{p_{b}^{(j)}, P_{a}^{(k)}\right\}=0, \\
\delta_{a}^{P, k} t_{(j)} & =\left\{t_{(j)}, P_{a}^{(k)}\right\}=0, \\
\delta_{a}^{P, k} E^{(j)} & =\left\{E^{(j)}, P_{a}^{(k)}\right\}=0 .
\end{aligned}
$$

Rotations and generalized rotations $J^{(k)}, k=0, \ldots, M$.

$$
\begin{aligned}
\delta_{a b}^{J, k} x_{(j)}^{c} & =\left\{x_{(j)}^{c}, J_{a b}^{(k)}\right\}=\delta_{a}^{c} x_{(j-k) b}-\delta_{b}^{c} x_{(j-k) a}, \\
\delta_{a b}^{J, k} p_{c}^{(j)} & =\left\{p_{c}^{(j)}, J_{a b}^{(k)}\right\}=-\delta_{b c} p_{a}^{(j+k)}+\delta_{a c} p_{b}^{(j+k)}, \\
\delta_{a b}^{J, k} t_{(j)} & =\left\{t_{(j)}, J_{a b}^{(k)}\right\}=0, \\
\delta_{a b}^{J, k} E^{(j)} & =\left\{E^{(j)}, J_{a b}^{(k)}\right\}=0 .
\end{aligned}
$$

The transformations of the $p^{(j)}$ and $E^{(j)}$ under boosts and rotations agree with those proposed in section 2 .

\section{Invariance of the constraints under the full set of transformations}

Here we show that the proposed constraints (2.2) are invariant (or weakly invariant in some cases) under the full set of transformations given by (4.7). Since for boosts and rotations the transformations given by the generators correspond to those of (2.12), for which the invariance of the constraints is proved in appendix A, we will deal here only with the rest of generators, that is $D, C, D^{(k)}, H^{(k)}$ and $P^{(k)}$. We will consider the transformations under the individual generators without multiplying by the corresponding parameters, using the results in appendix (B).

Dilatations $\boldsymbol{D}$. The terms which add up to form $\phi^{(k)}$ transform homogeneously under $D$ and one has

$$
\delta^{D} \phi^{(k)}=-\frac{2 M+2-2 k}{2 M+1} \phi_{(k)} \approx 0, \quad k=0,1, \ldots, M,
$$

where $\approx$ means equality on the constraints manifold, so that the constraints are weakly invariant under dilatations.

Expansions $C$. For expansions one has

$$
\delta^{C} \phi^{(k)}= \begin{cases}-2 t_{(0)} \phi^{(M)} & \text { for } k=0, \\ 0 & \text { for } k=1, \ldots, M\end{cases}
$$

and the constraints are also weakly invariant. 
Generalized dilatations $D^{(j)}, j=1, \ldots, M$. Under generalized dilatations one has

$$
\delta^{D, j} \phi^{(k)}= \begin{cases}-\frac{M+1-j-k}{M+1-j} \phi^{(k+j)} & \text { if } k+j \leq M \\ 0 & \text { otherwise }\end{cases}
$$

and we have weakly invariance.

Time shifts and space translations, $\boldsymbol{H}^{(j)}, \boldsymbol{P}^{(j)}, \boldsymbol{j}=\mathbf{0}, \ldots, \boldsymbol{M}$. The $P^{(l)}$ and $E^{(l)}$ are invariant under all these transformations, and hence so are the $\phi^{(k)}$,

$$
\delta^{H, j} \phi^{(k)}=\delta_{a}^{P, j} \phi^{(k)}=0, \quad j, k=0,1, \ldots, M .
$$

Open Access. This article is distributed under the terms of the Creative Commons Attribution License (CC-BY 4.0), which permits any use, distribution and reproduction in any medium, provided the original author(s) and source are credited.

\section{References}

[1] J. Gomis, A. Kleinschmidt, J. Palmkvist and P. Salgado-ReboLledó, Symmetries of post-Galilean expansions, Phys. Rev. Lett. 124 (2020) 081602 [arXiv:1910.13560] [INSPIRE].

[2] G. Dautcourt, Die Newtonische Gravitationstheorie als strenger Grenzfall der allgemeinen Relativitätstheorie (in German), Acta Phys. Pol. 65 (1964) 637.

[3] G. Dautcourt, On the newtonian limit of general relativity, Acta Phys. Pol. B 21 (1990) 755.

[4] L. Blanchet, T. Damour, B.R. Iyer, C.M. Will and A.G. Wiseman, Gravitational radiation damping of compact binary systems to second post-Newtonian order, Phys. Rev. Lett. 74 (1995) 3515 [gr-qc/9501027] [INSPIRE].

[5] A. Buonanno and T. Damour, Effective one-body approach to general relativistic two-body dynamics, Phys. Rev. D 59 (1999) 084006 [gr-qc/9811091] [InSPIRE].

[6] L. Blanchet, Gravitational radiation from post-Newtonian sources and inspiralling compact binaries, Living Rev. Rel. 17 (2014) 2 [arXiv:1310.1528] [INSPIRE].

[7] C.M. Will, The confrontation between general relativity and experiment, Living Rev. Rel. 17 (2014) 4 [arXiv: 1403.7377] [INSPIRE].

[8] T. Damour, P. Jaranowski and G. Schäfer, Fourth post-Newtonian effective one-body dynamics, Phys. Rev. D 91 (2015) 084024 [arXiv:1502.07245] [INSPIRE].

[9] A. Maheshwari, E. Nissimov and I. Todorov, Classical and quantum two-body problem in general relativity, Lett. Math. Phys. 5 (1981) 359 [arXiv:1611.02943] [INSPIRE].

[10] I. Todorov, Dynamics of relativistic point particles as a problem with constraints, JINR-E2-10125, (1976) [INSPIRE].

[11] A. Komar, Constraint formalism of classical mechanics, Phys. Rev. D 18 (1978) 1881 [INSPIRE].

[12] P. Droz-Vincent, Action-at-a-distance and relativistic wave equations for spinless quarks, Phys. Rev. D 19 (1979) 702 [INSPIRE].

[13] R. Giachetti and E. Sorace, Two-body Coulomb and gravitational interactions of relativistic point-like particles, Nuovo Cim. B $6 \mathbf{3}$ (1981) 666 [INSPIRE]. 
[14] V. Bargmann, On unitary ray representations of continuous groups, Annals Math. 59 (1954) 1 [INSPIRE].

[15] U. Niederer, The maximal kinematical invariance group of the free Schrödinger equation, Helv. Phys. Acta 45 (1972) 802 [inSPIRE].

[16] C.R. Hagen, Scale and conformal transformations in Galilean-covariant field theory, Phys. Rev. D 5 (1972) 377 [INSPIRE].

[17] G. Dautcourt, Post-Newtonian extension of the Newton-Cartan theory, Class. Quant. Grav. 14 (1997) A109 [gr-qc/9610036] [INSPIRE].

[18] M. Ergen, E. Hamamci and D. Van den Bleeken, Oddity in nonrelativistic, strong gravity, Eur. Phys. J. C 80 (2020) 563 [Erratum ibid. 80 (2020) 657] [arXiv: 2002. 02688] [INSPIRE].

[19] D. Van den Bleeken, Torsional Newton-Cartan gravity from the large $c$ expansion of general relativity, Class. Quant. Grav. 34 (2017) 185004 [arXiv:1703.03459] [INSPIRE].

[20] O. Khasanov and S. Kuperstein, (In)finite extensions of algebras from their Inonu-Wigner contractions, J. Phys. A 44 (2011) 475202 [arXiv:1103.3447] [INSPIRE].

[21] D. Hansen, J. Hartong and N.A. Obers, Gravity between Newton and Einstein, Int. J. Mod. Phys. D 28 (2019) 1944010 [arXiv: 1904.05706] [INSPIRE].

[22] J. Gomis, A. Kleinschmidt and J. Palmkvist, Galilean free Lie algebras, JHEP 09 (2019) 109 [arXiv: 1907.00410] [INSPIRE].

[23] J. Gomis, A. Kleinschmidt, J. Palmkvist and P. Salgado-Rebolledo, Galilean and Carollian expansions of relativistic symmetries, in preparation.

[24] M. Cariglia, G.W. Gibbons, J.W. van Holten, P.A. Horvathy and P.M. Zhang, Conformal Killing tensors and covariant Hamiltonian dynamics, J. Math. Phys. 55 (2014) 122702 [arXiv: 1404.3422] [INSPIRE].

[25] C. Batlle, J. Gomis and D. Not, Extended Galilean symmetries of non-relativistic strings, JHEP 02 (2017) 049 [arXiv: 1611.00026] [INSPIRE].

[26] C. Roger and J. Unterberger, The Schrödinger-Virasoro Lie group and algebra: from geometry to representation theory, Annales Henri Poincaré 7 (2006) 1477 [math-ph/0601050] [INSPIRE].

[27] M. Henkel, Local scale invariance and strongly anisotropic equilibrium critical systems, Phys. Rev. Lett. 78 (1997) 1940 [cond-mat/9610174] [INSPIRE].

[28] J. Negro, M.A. del Olmo and A. Rodríguez-Marco, Nonrelativistic conformal groups, J. Math. Phys. 38 (1997) 3786.

[29] C. Duval and P.A. Horvathy, Non-relativistic conformal symmetries and Newton-Cartan structures, J. Phys. A 42 (2009) 465206 [arXiv:0904.0531] [InSPIRE].

[30] C. Duval and P. Horvathy, Conformal Galilei groups, Veronese curves, and Newton-Hooke spacetimes, J. Phys. A 44 (2011) 335203 [arXiv:1104.1502] [INSPIRE].

[31] J. Lukierski, P.C. Stichel and W.J. Zakrzewski, Noncommutative planar particle dynamics with gauge interactions, Annals Phys. 306 (2003) 78 [hep-th/0207149] [INSPIRE].

[32] P.C. Stichel and W.J. Zakrzewski, Can cosmic acceleration be caused by exotic massless particles?, Phys. Rev. D 80 (2009) 083513 [arXiv:0904.1375] [InSPIRE]. 
[33] P.C. Stichel and W.J. Zakrzewski, Nonstandard approach to gravity for the dark sector of the universe, Entropy 15 (2013) 559 [arXiv:1301.4486] [INSPIRE].

[34] J. Gomis and K. Kamimura, Schrödinger equations for higher order non-relativistic particles and $N$-Galilean conformal symmetry, Phys. Rev. D 85 (2012) 045023 [arXiv:1109.3773] [INSPIRE].

[35] J.-M. Lévy-Leblond, Group-theoretical foundations of classical mechanics: the Lagrangian gauge problem, Commun. Math. Phys. 12 (1969) 64.

[36] G. Marmo, G. Morandi, A. Simoni and E.C.G. Sudarshan, Quasiinvariance and central extensions, Phys. Rev. D 37 (1988) 2196 [InSPIRE].

[37] Z.K. Silagadze, Relativistic mass and modern physics, Can. J. Phys. 92 (2014) 1643 [arXiv:1103.6281] [INSPIRE].

[38] J. Sucher, Relativistic invariance and the square-root Klein-Gordon equation, J. Math. Phys. 4 (1963) 17.

[39] P.P. Fiziev, Relativistic Hamiltonian with square root in the path integral formalism, Theor. Math. Phys. 62 (1985) 123 [Teor. Mat. Fiz. 62 (1985) 186] [inSPIRE].

[40] J. Brugues, J. Gomis and K. Kamimura, Newton-Hooke algebras, non-relativistic branes and generalized pp-wave metrics, Phys. Rev. D 73 (2006) 085011 [hep-th/0603023] [INSPIRE]. 